\title{
SPECIES DIVERSITY AND VEGETATION STRUCTURE FROM DIFFERENT CLIMATIC ZONES OF TEHSIL HARIGHEL, BAGH, AZAD KASMIR, PAKISTAN ANALYSED THROUGH MULTIVARIATE TECHNIQUES
}

\author{
AKHLAQ, R. ${ }^{1}$ - AMJAD, M. S. ${ }^{*}{ }^{*}$-QASEEM, M. F. ${ }^{2}$-FATIMA, S. ${ }^{3}$ - CHAUDHARI, S. K. ${ }^{4}$ - KHAN, A. M. ${ }^{2}-$ \\ KHAN, S. ${ }^{1}-$ MALIK, N.Z. ${ }^{5}-$ GARDAZI, S. M. H. ${ }^{1}-$ BIBI, A. ${ }^{1}-$ SABAOON $^{2}$ \\ ${ }^{I}$ Department of Botany, Women University of Azad Jammu \& Kashmir, Bagh, Pakistan \\ ${ }^{2}$ Department of Botany, PMAS-University of Arid Agriculture, Rawalpindi, Pakistan \\ ${ }^{3}$ Department of Botany, Hafiz Hayat Campus, University of Gujarat, Pakistan \\ ${ }^{4}$ Department of Botany, The University of Lahore, Sargodha Campus, Pakistan \\ ${ }^{5}$ Department of Botany, Mirpur University of Science \& Technology, AJK, Pakistan \\ *Corresponding author \\ e-mail: malikshoaib1165@yahoo.com \\ (Received $2^{\text {nd }}$ Jun 2018; accepted $17^{\text {th }}$ Jul 2018)
}

\begin{abstract}
The phytosociological survey of western Himalayan subtropical and moist temperate forests of Tehsil Harighel, District Bagh Azad Jammu and Kashmir, Pakistan was carried out during 2015-2017. Sampling was done by using random stratified sampling technique at 12 different sites. Based on importance value twelve plant communities were recognized. They were merged into three plant associations using cluster analysis and Detrended correspondence analysis viz. Olea -Dodonea Micromeria association, Pinus- Oxalis-Dactylis association and Pinus-Diospyros -Myrsine association. The average number of species per site varied between 17-48, Species richness 3.00 to7.01; Shannon Diversity 2.49 to 3.82 ; Evenness 0.46 to 0.72 and Equitability 0.73 to 0.91 . Canonical Correspondence Analysis (CCA) revealed that altitude and edaphic characteristics like potassium, organic matter, saturation, electrical conductivity and $\mathrm{pH}$ play a significant role in controlling the distribution pattern of plant species. Low value of species diversity and associated components clearly reflect that forest structure is deteriorated in the investigated area with poor regeneration potential. Therefore, immediate conservation measures by integrating local populations' perceptions are urgently recommended.
\end{abstract}

Keywords: environmental gradient, cluster analysis, richness, DCA, CCA

\section{Introduction}

Vegetation structure, species composition, diversity and richness pattern, maturity value are the important ecological characteristics that are highly correlated with anthropogenic and environmental variables (Gairola et al., 2008; Shaheen et al., 2011a; Ahmad et al., 2013; Amjad et al., 2014a, b; Ilyas et al., 2015). Vegetation is the distinct physiognomic unit whose structure can be clearly differentiated from other such unit (Hussain and Illahi, 1991). Vegetation, soil and climate are interrelated with each other. The variation in any one of these components may bring change in the associated component (Kent, 2012; Amjad et al., 2014b; Ilyas et al., 2015). Geographical or topographical factors like altitude and aspect play critical role in structuring vegetation (Ellenberg, 1996; Gallardo-Cruz et al., 2009; Scherrer and Korner, 2010; Shaheen et al., 2011b). Vegetation is the chief component of environment in majority of habitat which 
facilitates the ecosystem services and biodiversity (Gardner et al., 2009). Therefore assessment of vegetation structure is the main indicator in conservation and ecosystem management.

Species diversity is a function of the number of species present in a given area and reflects the productivity and health of ecosystem (Ruiz et al., 2008). The most easily interpretable indicator of diversity is species richness which is controlled by a complex of environmental variables (Whittaker, 1977; Shrestha and Jha, 2009) mainly altitude, aspect and moisture (Vetaas, 2000; Schuster and Diekmann, 2005) The precise diversity measurement is helpful in understanding the various phenomena involved in organization and development of plant communities (Shoukat et al., 1978; Malik and Malik, 2012). The anthropogenic pressure in the form of deforestation, overgrazing and fuel wood extraction can be clearly reflected from low species diversity and richness in the area. Thus vegetation structure information along with species biodiversity assessment is a prerequisite for biodiversity conservation and ecosystem management (Willoughby and Alexander, 2000, 2005).

The computer-based multivariate statistical techniques helps ecologists in structuring large data sets and analyzing impact of environmental factors on distribution pattern of plant species (Bergmeier, 2002; Anderson et al., 2006). Such techniques reduce the complexity of data by classifying vegetation and relating the results to abiotc (environmental) components (Dufrene and Legendre, 1997; McCune and Mefford, 1999; Ter Braak and Prentice, 1988). Various multivariate techniques such as Two way Indicator species analysis, Cluster analysis, Canonical correspondence analysis and Detrended correspondence analysis have been extensively employed by ecologists recognize the environmental gradient among vegetation structure and species diversity in mountainous ecosystems (Daubenmire, 1968; Vetaas and Grytnes, 2002; Tavili and Jafari, 2009).

Azad Jammu \& Kashmir, Pakistan harboured diverse climate, soil type and rich diversity. Various studies have been conducted previously to analyze vegetation in different part of Azad Jammu \& Kashmir (Siddiqui et al., 2010; Shaheen et al., 2011a; Malik and Malik, 2012; Amjad et al., 2014a,b; Bokhari et al., 2016; Amjad et al., 2017). However many potentially diverse remote areas like Harigal were not still explored by the ecologist particularly using advanced phytosociological techniques. Moreover previous studies are restricted to floristic inventory and quantitative attributes and lack novelty in term of multivariate analysis. Therefore present study was designed to analyze the variation among species diversity and vegetation structure along the environmental gradient using multivariate approach. The findings of current research work will be helpful for designing effective conservation strategies for sustainable management of plant resources of area particular for minimizing anthropogenic pressure.

\section{Materials and methods}

\section{Study area}

Tehsil Harighel of District Bagh, Azad Jammu \& Kashmir, Pakistan is located in western Himalayan foot hills of Pir-Panjal range between latitude $33^{\circ} 54^{\prime}-34^{\circ} 08^{\prime} \mathrm{N}$ and longitude $73^{\circ} 01^{\prime}-73^{\circ} 38^{\prime}$ E longitude. The altitude of area varies between $980 \mathrm{~m}$ to $2052 \mathrm{~m}$. Total area of District Bagh is $1368 \mathrm{~km}^{2}$ with $54.78 \%$ under forest cover. Population is 0.434 million (Anonymous 2007). The climate of area varies from 
subtropical humid to temperate type at various elevations with mean annual precipitation of $1500 \mathrm{~mm}$. The average annual temperature is $21{ }^{\circ} \mathrm{C}$ with maximum up to $40{ }^{\circ} \mathrm{C}$ during July and minimum up to $2{ }^{\circ} \mathrm{C}$ during January (Anonymous, 2009; Shaheen et al., 2011a).

\section{Collection and identification of plant specimen}

Three plant specimens of each species were collected. The specimens were carefully dried and mounted on herbarium sheet. Different floras and monograph of various areas of Pakistan were used for Identification of specimens. The identified specimens were confirmed at AJ\&K Medicinal and Aromatic Plant Herbarium PARC, Pakistan. International Plant Name Index (IPNI) was used to obtain correct botanical name. The identified specimens were deposited in Herbarium of Women University of Azad Jammu \& Kashmir Bagh.

\section{Field sampling}

Field surveys were carried out during 2014-2016 following specific locality procedure. Twelve sites were selected based on altitude, aspect and physiognomy (Fig. 1). The altitude and geographical coordinates of each site was determined by using GPS and aspect by using Suunto Tandem survey master compass. Detailed stratified sampling was done using quadrat method. The quadrat size was $1 \times 1 \mathrm{~m}^{2}, 5 \times 5 \mathrm{~m}^{2}$ and $10 \times 10 \mathrm{~m}^{2}$ for herbs, shrubs and trees respectively. Composition and abundance data of plant species were recorded from each quadrat on prepared Excel data sheets.

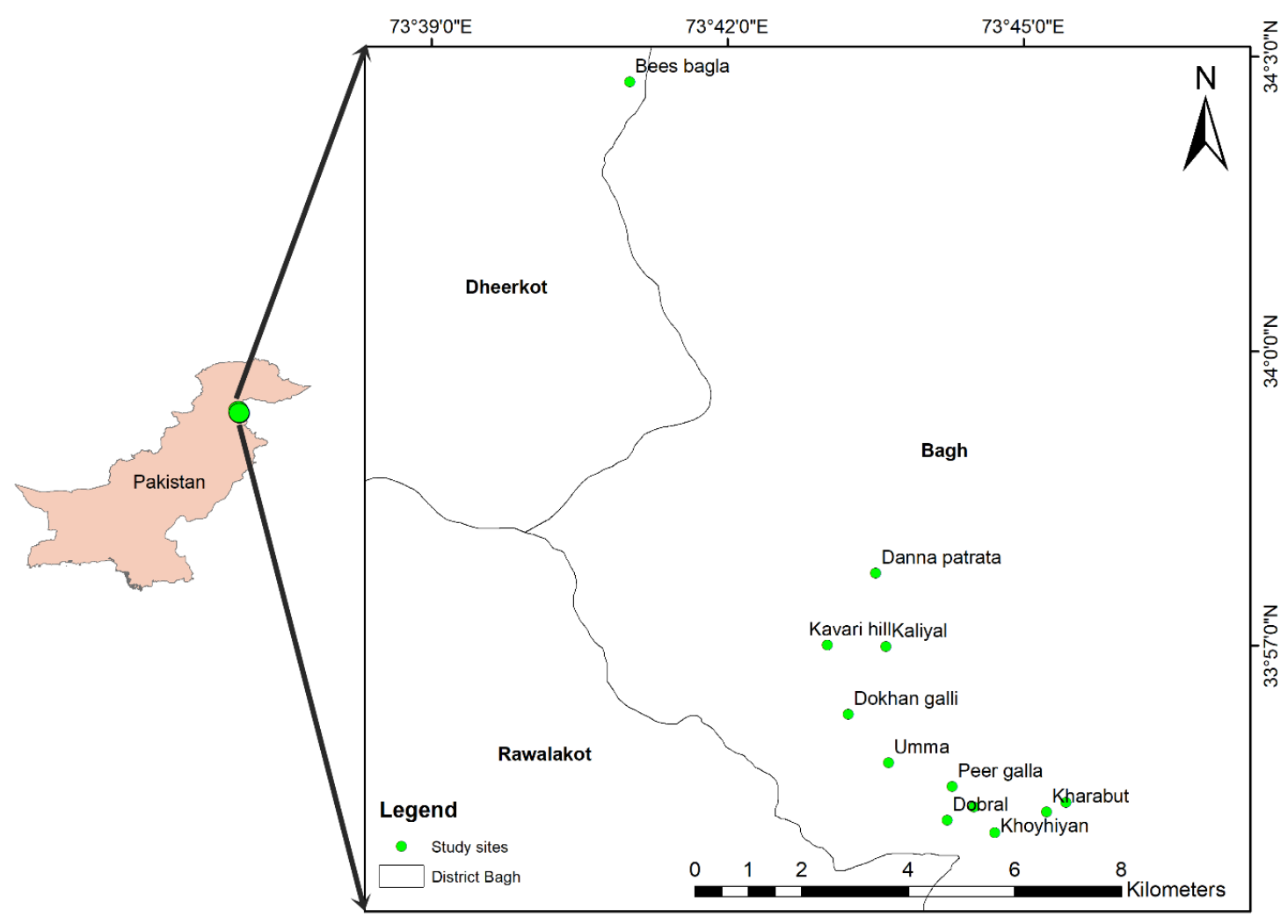

Figure 1. Map showing location of different study sites 
Three soil samples were collected from each site at depth of $15 \mathrm{~cm}$ and mixed to make composite. Different physico-chemical properties of soil like texture, $\mathrm{pH}$, organic matter, electrical conductivity, saturation, $\%$ of phosphorous, potassium and calcium carbonate were determined in Pakistan Soil and Water Testing Laboratory Rawalpindi using standard methods (Jackson, 1962; Hussain, 1989).

\section{Data storage and analysis}

The importance value of each plant species was recorded following Curtis and McIntosh (1950) by using Equation 1:

$$
I V I=R \cdot D+R \cdot F+R \cdot C .
$$

where IVI = importance value index, R.D = relative density, R.F = relative frequency, and R.C. $=$ relative cover.

Species diversity was calculated by following Shannon (1949) by using Equation 2:

$$
H=\Sigma\left(\frac{n}{\mathbb{N}} \times \frac{\ln (n)}{\ln (\mathbb{N})}\right)
$$

where $\mathrm{H}=$ species diversity, $\mathrm{n}=$ number of individual of its species, $\mathrm{N}=$ total number of individual of all species.

Species richness was calculated by following Margalef (1958) using Equation 3:

$$
R=\frac{s-1}{\ln (M)}
$$

where $\mathrm{R}=$ species richness, $\mathrm{N}=$ total number of individual of all species.

Equitability was calculated by following Sheldon (1969) using Equation 4:

$$
E=\frac{H}{\ln (\Omega)}
$$

where $\mathrm{H}=$ Diversity, $\mathrm{S}=$ total number of species.

Evenness was calculated by following Gibson's evenness index using Equation 5:

$$
G=\frac{e H}{s}
$$

where, $\mathrm{H}=$ Diversity, $\mathrm{S}=$ total number of species.

Species maturity was calculated by following Pichi-Sermollis (1948) using Equation 6:

$$
M=\frac{F}{s}
$$

where, $\mathrm{M}=$ Species maturity, $\mathrm{F}=$ total frequency, $\mathrm{S}=$ total species number.

Species composition and abundance data of 158 species and 7 environmental variables from 390 quadrats at twelve different sampling sites (390 quadrats) were processed in MS Excel in accordance with the PCORD V.5 and CANACOO V.5 requirements. The grouping of plant species was carried out by using cluster analysis in PC ORD software (McCune and Grace, 2002; McCune and Mefford, 2005). Wards 


$$
-5197 \text { - }
$$

method was used as linkage method to group the same stands. These associations were documented at three level of division on the bases of dendrogram. The vegetation and environmental data was further subjected to ordination analysis using CANACOO version 5.00 (TerBraak and Smilauer, 2002). DCA and CCA analysis was carried out to verify the faithfulness of grouping/association, check the ecological gradient of plant association and to find the vegetation environmental relation. The explanatory power (strength) of different environmental factors was checked by Monte Carlo Permutation test (reduced model, 4999 permutations).

\section{Results}

Based on cluster analysis and DCA, 158 plant species and 12 sites were grouped into three associations (Fig. 2) which are as follows:

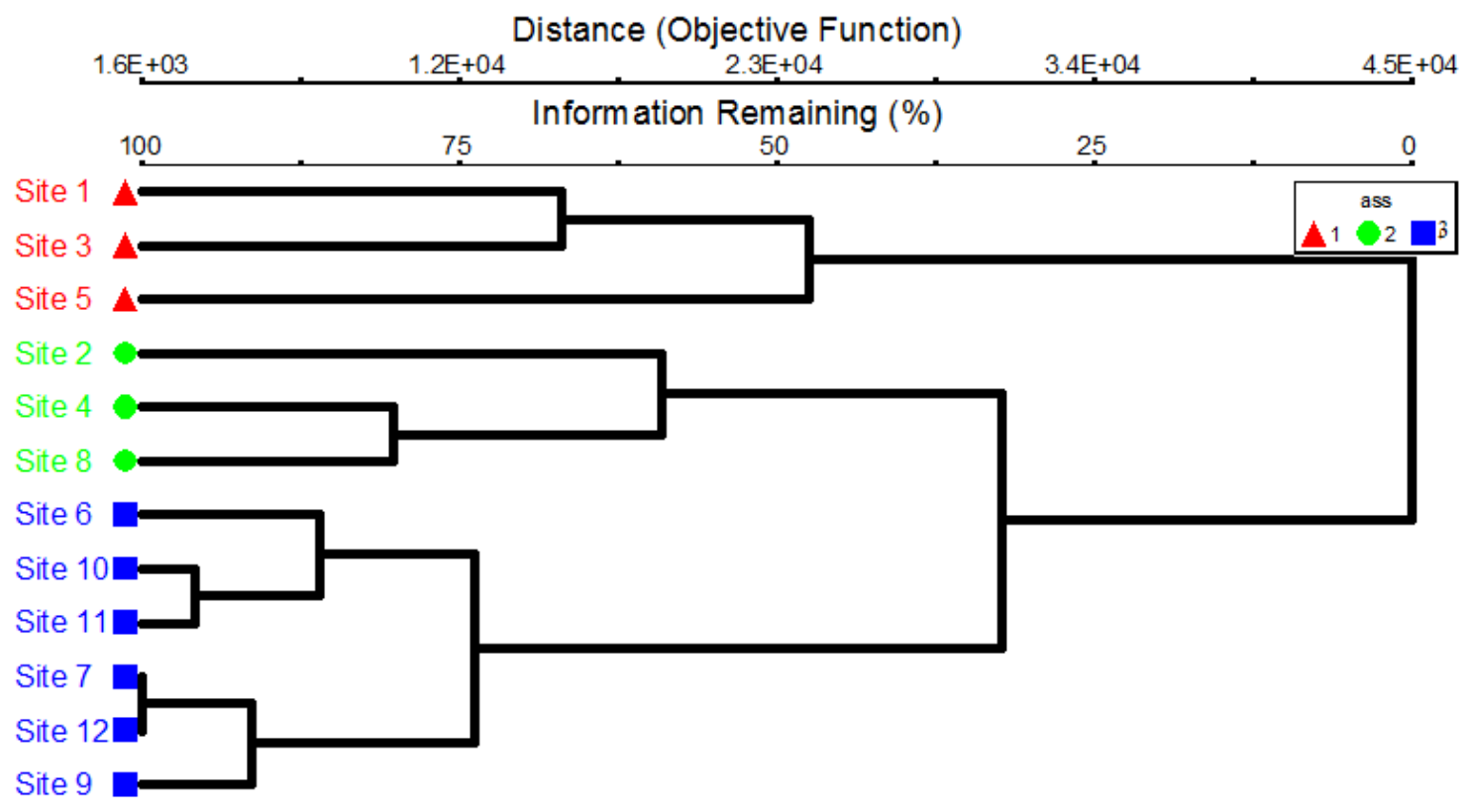

$$
\begin{aligned}
& \text { Key: } \\
& \boldsymbol{\Delta}=\text { Sites of Olea-Dodonea-Micromeria association } \\
& \text { - = Sites of Pinus-Oxalis-Dactylis association } \\
& \text { - Sites of Pinus-Diospyrus -Myrsine association }
\end{aligned}
$$

Figure 2. Cluster analysis dendrogram representing three plant associations

\section{Olea-Dodonea-Micromeria (A) association}

This association harboured at an altitudinal range of 980-1165 m. The association comprised of three communities having 51 species which include 3 trees, 15 shrubs and 33 herbs. The dominant species were Olea ferruginea $(\mathrm{I} . \mathrm{V}=60.02)$, Dodoneae viscosa $(\mathrm{IV}=27.21)$ and Micromeria biflora (I9.92). Whereas Berberis lycium, Maytenus nemorosa and Ficus palmata were associated plant species (Table 1). Soil was loamy with average $\mathrm{pH}$ of 7.36 . Soil saturation was $40 \%$; electrical conductivity $0.83 \mu \mathrm{s} / \mathrm{cm}$; organic matter $0.73 \%$; phosphorus $3.66 \mathrm{mgKg}^{-1}$ and potassium $106.66 \mathrm{mgKg}^{-1}$. 


\section{Pinus-Oxalis-Dactylis (B) association}

This association lies in between 1079 and $1658 \mathrm{~m}$ and consisted of 3 plant communities having 79 Plant species which include 8 trees, 15 shrubs and 56 herbs. Pinus roxburghii (I. V = 33.65), Oxalis spiralis $(\mathrm{I} . \mathrm{V}=12.64)$ and Dactylis glomerata (I. V = 11.81) were dominant. Whereas Myrsine Africana and Indigofera linifolia were associated plant species (Table 1). Soil texture was loamy with average $\mathrm{pH}$ 7.17. Soil saturation 39.66\%; electrical conductivity $0.70 \mu \mathrm{s} / \mathrm{cm}$; organic matter $0.76 \%$; phosphorus $4.4 \mathrm{mg} \mathrm{kg}^{-1}$ and potassium $120 \mathrm{mg} \mathrm{kg}^{-1}$.

\section{Pinus-Diospyrus-Myrsine (C) association}

This association was recorded at an altitude of 1450-20520 m having 6 Plant communities having 110 species. Pinus wallichiana (I. V = 40.13), Diospyrus lotus (I. $\mathrm{V}=11.84$ ) and Myrsine Africana (I. V=9.78) were dominant. Quercus dilitata, Berberis lycium, Sarcococca saligna and Vibernum grandiflorum were the associated plant species (Table 1). Soil was loamy with average $\mathrm{pH}$ of 7.23. Soil saturation was 40.05\%; electrical conductivity $0.64 \mu \mathrm{s} / \mathrm{cm}$; organic matter 0.81 ; phosphorus $5.33 \mathrm{mgKg}^{-1}$ and potassium $116.66 \mathrm{mg} \mathrm{kg}^{-1}$.

Table 1. Mean relative importance value of species in three associations recorded by normal cluster analysis during monsoon 2016 from Tehsil Harighel

\begin{tabular}{c|c|c|c|c}
\hline Species name & Abbrev. & $\begin{array}{c}\text { Association } \\
\text { A }\end{array}$ & $\begin{array}{c}\text { Association } \\
\text { B }\end{array}$ & $\begin{array}{c}\text { Association } \\
\text { C }\end{array}$ \\
\hline Olea ferruginea Royle. & Ole fer & 60.02 & 9.01 & 3.55 \\
\hline Pinus roxburghii Sarg. & Pin rox & 9.71 & 33.65 & 2.32 \\
\hline Acacia nilotica (L.) Wild. ex Delile & Aca nil & 2.47 & 0 & 1.47 \\
\hline Pyrus pashia Buch.-Ham. ex D. Don & Pyr pas & 0 & 6.21 & 4.97 \\
\hline Diospyros lotus L. & Dio lot & 0 & 5.16 & 11.84 \\
\hline Pinus wallichiana L. & Pin wal & 0 & 8.22 & 40.13 \\
\hline Ailanthus altissima (Mill.) Swingle & Ail alt & 0 & 4.09 & 1.04 \\
\hline Quercus dilatata Lindl. & Que dil & 0 & 0 & 9.52 \\
\hline Dalbergia sissoo Roxb. ex DC & Dal sis & 0 & 0 & 0.39 \\
\hline Broussonetia papyrifera (L.) L Her. Ex Vent. & Bro pap & 0 & 0 & 0.88 \\
\hline Juglans regia L. & Jug reg & 0 & 0 & 1.56 \\
\hline Aesculus indica (Wall. ex Cambess.) Hook. & Aes ind & 0 & 0 & 1.00 \\
\hline Mimosa pudica L. & Mim pud & 0 & 0 & 0.64 \\
\hline Quercus incana Roxb. & Que ina & 0 & 1.90 & 4.63 \\
\hline Salix nigra Marshall & Sal nig & 0 & 2.40 & 0 \\
\hline Bauhinia variegate L. & Bau var & 0 & 0 & 1.08 \\
\hline Dodonaea viscosa (L.) Jacq. & Dod vis & 27.21 & 0 & 0 \\
\hline Maytenus nemorosa Marais & May nem & 10.83 & 0 & 0 \\
\hline Myrsine africana L. & Myr afr & 8.55 & 11.09 & 9.78 \\
\hline Berberis lycium Royle. & Ber lyc & 11.80 & 5.98 & 9.23 \\
\hline Cotoneaster racemiflorae Pojark. & Cot rac & 4.32 & 2.68 & 1.65 \\
\hline Zanthozylum alatum Roxb. & Zan ala & 5.12 & 4.07 & 2.43 \\
\hline
\end{tabular}




\begin{tabular}{|c|c|c|c|c|}
\hline Nerium oleander L. & Ner ole & 6.46 & 0 & 0.77 \\
\hline Rubus fruticosus $\mathrm{L}$. & Rub fru & 0 & 0 & 0.72 \\
\hline Indigofera linifolia (L. f.) Retz & Ind lin & 0 & 10.12 & 3.76 \\
\hline Viburnum grandiflorum Wall. ex DC. & Vib gra & 0 & 0 & 8.70 \\
\hline Rosa brunonii Lindl. & Ros bru & 1.30 & 5.42 & 4.64 \\
\hline Ricinus communis L. & Ric com & 0 & 2.03 & 0 \\
\hline Wikstroemia canescens Wall. ex Meisn. & Wik can & 1.61 & 6.53 & 5.90 \\
\hline Debregearsia salcifolia & Deb sal & 0 & 6.38 & 0.75 \\
\hline Sarcococca saligna (D. Don) Müll. Arg. & Sar sal & 0 & 3.20 & 8.18 \\
\hline Ziziphus mauritiana Lams. & Ziz mau & 2.69 & 0 & 0 \\
\hline Adhatoda zeylanica Medik. & Adh zey & 0 & 0 & 0.81 \\
\hline Desmodium elegans Schltdl. & Des ele & 0 & 0 & 0.49 \\
\hline Petrorhagia saxifraga (L.) Link & Pet sax & 0 & 0 & 0.40 \\
\hline Rubus niveus Wall. ex G. Don & Rub niv & 0 & 0 & 1.14 \\
\hline Jasminum grandiflorum L. & Jas gra & 0 & 0 & 1.28 \\
\hline Ficus palmata Forssk. & Fic pal & 10.24 & 4.59 & 3.74 \\
\hline Punica granatum $\mathrm{L}$ & Pun gra & 4.98 & 2.49 & 2.44 \\
\hline Rubus ellipticus $\mathrm{Sm}$. & Rub ell & 0 & 8.70 & 1.28 \\
\hline Elaeagnus umbellate & Ela umb & 0 & 0 & 2.14 \\
\hline Oteostagia limbata (Benth.) Boiss. & Ote lim & 3.83 & 0 & 0 \\
\hline Rumex hastatus D. Don & Rum has & 1.10 & 2.61 & 0.97 \\
\hline Rhus catinus Scop. & Rhu cat & 1.66 & 2.03 & 1.07 \\
\hline Machilus odoratissimus Nees & Mac odo & 0 & 2.52 & 2.41 \\
\hline Jasminum humile L. & Jas hum & 0 & 0 & 0.49 \\
\hline Asplenium adiantum-nigrum $\mathrm{L}$. & Asp adi & 0 & 2.31 & 0.89 \\
\hline Lespedeza juncea (L. f.) Pers. & Les jun & 4.36 & 3.05 & 0 \\
\hline Senecio amplectens A. Gray & Sen amp & 0 & 2.33 & 0.47 \\
\hline Adiantum caudatum $\mathrm{L}$. & Adi cau & 0 & 1.08 & 2.90 \\
\hline Clinopodium vulgare L. & Cli vul & 0 & 0.90 & 0 \\
\hline Scutellaria ovata Hill & Scu ova & 5.83 & 0.96 & 0 \\
\hline Eulaliopsis binata Retz. & Eul bin & 5.48 & 1.18 & 0 \\
\hline Adiantum incisum Forssk. & Adi inc & 0 & 0.30 & 0 \\
\hline Oenothera rosea L Her. ex Aiton & Oen ros & 0 & 0.47 & 6.70 \\
\hline Thalictrum Spp. & Tal spp & 0 & 1.06 & 0.73 \\
\hline Androsace rotundifolia Hardw. & And rot & 1.21 & 0.51 & 0.78 \\
\hline Micromeria biflora (Buch.-Ham. ex D. Don) Benth. & Mic bif & 19.92 & 7.48 & 7.11 \\
\hline Poa pratensis $L$ & Poa pra & 0 & 0 & 1.26 \\
\hline Ajuga parviflora Benth. & Aju par & 1.38 & 0 & 1.64 \\
\hline Setaria pumila (Poir.) Roem. \& Schult. & Set pum & 5.47 & 0 & 0 \\
\hline Lepidium ruderale $\mathrm{L}$. & Lep rud & 0 & 5.57 & 0 \\
\hline Oxalis spiralis Ruiz \& Pav. ex G. Don & Oxa spi & 0 & 12.64 & 1.77 \\
\hline Fragaria visca L. & Fra vis & 0 & 0.62 & 4.60 \\
\hline Sonchus arvensis L. & Son arv & 0 & 0.94 & 1.29 \\
\hline
\end{tabular}




\begin{tabular}{|c|c|c|c|c|}
\hline Clinopodium umbrosum (M. Bieb.) K. Koch & Cli umb & 0 & 2.05 & 0 \\
\hline Oxalis corniculata $\mathrm{L}$. & Oxa cor & 0 & 7.43 & 0 \\
\hline Pteris cretica $\mathrm{L}$. & Pte cre & 0 & 4.83 & 2.80 \\
\hline Origanum vulgare $L$. & Ori vul & 0 & 0 & 0.23 \\
\hline Dryopteris filix-mas (L.) Schott & Dry fil & 7.03 & 2.78 & 0 \\
\hline Dactylis glomerata L. & Dac glo & 0 & 11.81 & 0.54 \\
\hline Bidens biternata (Lour.) Merr. \& Sherff & Bid bit & 4.16 & 0 & 0 \\
\hline Themeda anathera (Nees ex Steud.) Hack & The ana & 6.78 & 8.47 & 0 \\
\hline Conyza canadensis (L.) Cronquist & Con can & 1.49 & 0 & 1.07 \\
\hline Ipomoea purpurea (L.) Lam. & Ipo pur & 4.21 & 0 & 0.73 \\
\hline Taraxacum officinale L. & Tar ofi & 2.73 & 0.28 & 2.40 \\
\hline Veronica laxa (Benth.) & Ver lax & 2.89 & 4.85 & 0.87 \\
\hline Carpesium abrotanoides $\mathrm{L}$. & Car abr & 2.25 & 0 & 0 \\
\hline Crotalaria juncea $L$. & Cro jun & 4.95 & 0 & 0 \\
\hline Agrostis stolonifera L. & Agr sto & 3.40 & 0 & 0.58 \\
\hline Gerbera gossypina (Royle) Beauverd & Ger gos & 0 & 4.78 & 1.81 \\
\hline Verbascum thapsun $\mathrm{L}$. & Ver tha & 0.07 & 0.33 & 0 \\
\hline Geranium ocellatum Cambess & Ger oce & 0 & 0.62 & 0 \\
\hline Cynoglosum lanceolatum Forssk & Cyn lan & 0 & 0.04 & 0.45 \\
\hline Galium aparine $\mathrm{L}$. & Gal apa & 0 & 2.25 & 6.19 \\
\hline Vicia sativa Guss. & Vic sat & 0 & 0.74 & 1.69 \\
\hline Cynodon dactylon (L.) Pers. & Cyn dac & 0 & 2.85 & 2.94 \\
\hline Pteris umbrosa $\mathrm{R}$. Br. & Pte umb & 0.73 & 0 & 0 \\
\hline Cichorium intybus L. & Cic int & 0 & 0.97 & 0 \\
\hline Prunella vulgaris L. & Pru vul & 0 & 0.99 & 1.95 \\
\hline Viola canescens Wall. & Vio can & 0 & 1.44 & 0 \\
\hline Euphorbia helioscopia L. & Eup hel & 0 & 6.98 & 0 \\
\hline Arundo donax L. & Aru don & 0 & 0.87 & 0 \\
\hline Onopordum acanthium L. & Ono aca & 0 & 1.65 & 0 \\
\hline Mentha arvensis L. & Men arv & 0 & 2.54 & 1.23 \\
\hline Mentha longifolia $\mathrm{L}$. & Men lon & 0 & 1.49 & 1.69 \\
\hline Epipactis helleborine (L.) Crantz & Epi hel & 0 & 3.57 & 0 \\
\hline Cyprus niveus & Cyp niv & 0 & 1.53 & 1.46 \\
\hline Silybum marianum (L.) Gaertn. & Sil mar & 0.40 & 0 & 0.43 \\
\hline Euphorbia hirta & Eup hir & 0.07 & 0 & 1.77 \\
\hline Aegopodium podagraria $L$. & Aeg pod & 12.08 & 0 & 0 \\
\hline Valerianella szovitsiana Fisch. \& C.A. Mey. & Val szo & 3.38 & 0 & 0 \\
\hline Linum spp. & Lin spp & 0 & 0 & 1.69 \\
\hline Trichodesma indicum (L.) Lehm & Tri ind & 6.45 & 0 & 0 \\
\hline Astragalus leucocephalus Graham ex Benth. & Ast leu & 1.64 & 0 & 0 \\
\hline Salvia lanata Roxb. & Sal lan & 2.21 & 0 & 0 \\
\hline Trifolium repens $\mathrm{L}$. & Tri rep & 0 & 0 & 5.16 \\
\hline Polygala abyssinica R. Br. ex Fresen & Pol aby & 3.88 & 0 & 0 \\
\hline
\end{tabular}




\begin{tabular}{|c|c|c|c|c|}
\hline Spirace canescans & Spi can & 2.56 & 0 & 0 \\
\hline Plantago major $\mathrm{L}$. & Pla maj & 0 & 0 & 4.77 \\
\hline Lactuca floridana (L.) Gaertn. & Lac flo & 0 & 0 & 0.53 \\
\hline Adiantum capillus-veneris $\mathrm{L}$. & Adi cap & 0 & 0 & 1.98 \\
\hline Ranunculus arvensis $\mathrm{L}$ & Ran arv & 0 & 2.11 & 2.13 \\
\hline Ranunculus sceleratus L. & Ran sce & 0 & 0 & 4.65 \\
\hline Hedera nepalensis $K$. Koch & Hed nep & 3.00 & 0 & 2.98 \\
\hline Pennisetum orientale Rich. & Pen ori & 12.48 & 1.17 & 1.01 \\
\hline Cannabis sativa $\mathrm{L}$. & Can sat & 0 & 4.32 & 0 \\
\hline Plantago lanceolata $\mathrm{L}$. & Pla lan & 0 & 3.44 & 2.72 \\
\hline Plectranthus spp & Ple spp & 0 & 1.58 & 0 \\
\hline Solanum nigrum $\mathrm{L}$. & Sol nig & 0 & 2.43 & 0 \\
\hline Lathyrus aphaca $\mathrm{L}$. & Lat aph & 0 & 1.25 & 0 \\
\hline Juncus articulates & Jun art & 0 & 0 & 1.03 \\
\hline Cirsium vulgare (Savi) Ten & Cir vul & 0 & 0 & 0.41 \\
\hline Dicliptera bupleuroides Nees & Dic bup & 0 & 0 & 1.46 \\
\hline Phlomis bracteosa Royle ex Benth. & $\mathrm{Phl}$ bra & 0 & 0 & 0.63 \\
\hline Polygonatum geminiflorum Decne. & Pol gem & 0 & 0 & 1.77 \\
\hline Dryopterious odantoloma & Dry oda & 0 & 0 & 0.95 \\
\hline Thymus linearis Benth. & Thy lin & 0 & 0 & 1.79 \\
\hline Achillea millefolium $\mathrm{L}$. & Ach mil & 0 & 0 & 0.94 \\
\hline Vicia monantha Retz. & Vic mon & 0 & 0 & 0.76 \\
\hline Artemisia vulgaris $L$. & Art vul & 0 & 0 & 2.09 \\
\hline Astrogates condolleances & Ast con & 0 & 0 & 0.56 \\
\hline Adiantum tenerum $\mathrm{Sw}$. & Adi ten & 0 & 0 & 0.93 \\
\hline Anaphalis triplinervis (Sims) C.B. Clarke & Ana tri & 0 & 0 & 0.33 \\
\hline Onopordum acanthum & Ono aca & 0 & 1.65 & 0 \\
\hline Urtica dioca $\mathrm{L}$ & Urt dio & 0 & 0 & 0.85 \\
\hline Scutellaria chamaedrifolia Hedge \& Paton & Scu cha & 0 & 0 & 0.10 \\
\hline Serratula praealta $\mathrm{L}$. & Ser pra & 0 & 0 & 0.59 \\
\hline Clematis grata Wall. & Cle gra & 0 & 0 & 0.64 \\
\hline Silene conoidea $\mathrm{L}$. & Sil con & 0 & 0 & 0.36 \\
\hline Convolvulus arvensis $\mathrm{L}$. & Con arv & 0 & 0 & 0.67 \\
\hline Anagalis arvensis L. & Ana arv & 1.17 & 0 & 0.47 \\
\hline Barleria cristata $\mathrm{L}$. & Bar cri & 3.24 & 0 & 0 \\
\hline Stellaria media (L.) Cirillo & Ste med & 0 & 0.83 & 0 \\
\hline Rumex dantatus $\mathrm{L}$ & Rum dan & 2.29 & 0 & 0.23 \\
\hline Fragaria nubicola & Fra nub & 0 & 0 & 4.61 \\
\hline Duchesnia indica (Andr.) Focke & Dus ind & 0 & 2.86 & 0 \\
\hline Adiantum venustum D. Don & Adi ven & 0 & 0 & 0.76 \\
\hline Hedera helix K.Koch & Hed hel & 0 & 2.56 & 1.14 \\
\hline Brachiaria reptans (L.) C.A. Gardner \& C.E. Hubb. & Bra rep & 0 & 0 & 0.09 \\
\hline Pteris cheltron Andr. & Pte che & 0 & 0 & 1.15 \\
\hline
\end{tabular}




\begin{tabular}{c|c|c|c|c}
\hline Vincetoxicum hirundinaria Medik. & Vin hir & 0 & 0 & 0.69 \\
\hline Galium asprellum Michx. & Gal asp & 0 & 4.39 & 2.12 \\
\hline Galium asperuloides Edgew. S & Gal aspe & 0 & 0 & 1.56 \\
\hline Galium elegan Blocki & Gal ele & 0 & 0.79 & 0 \\
\hline Clematis barbellateEdgew. & Cle bar & 0 & 0.90 & 0 \\
\hline Medicago polymorpha $\mathrm{L}$. & Med pol & 0 & 0 & 0.72 \\
\hline
\end{tabular}

$\mathrm{A}=$ Olea-Dodonea-Micromeria association; $\mathrm{B}=$ Pinus-Oxalis-Dactylis association; $\mathrm{C}=$ PinusDiospyrus-Myrsine association

\section{Detrended correspondence analysis}

Detrended Correspondence analysis is the indirect gradient analysis which applied here to highlight the ecological gradients controlling the spatial variations among the plant species. Sum of all variance or eigen values was 3.93. The first Eigen value was proven to be quite high (0.57) which reflect strong gradient strength in species distribution patterning along this DCA axis. The DCA diagram explains altitudinal, aspect and environmental gradient. The sites and species of lower altitude, northern aspect and mesic habitat (Pinus-Diospyros-Myrsine association) were positioned on right side of diagram whereas site and species of higher altitude southern aspect and xeric habitat (Olea-Dodonea-Micromeria association) were positioned on left side of diagram (Figs. 3 and 4).

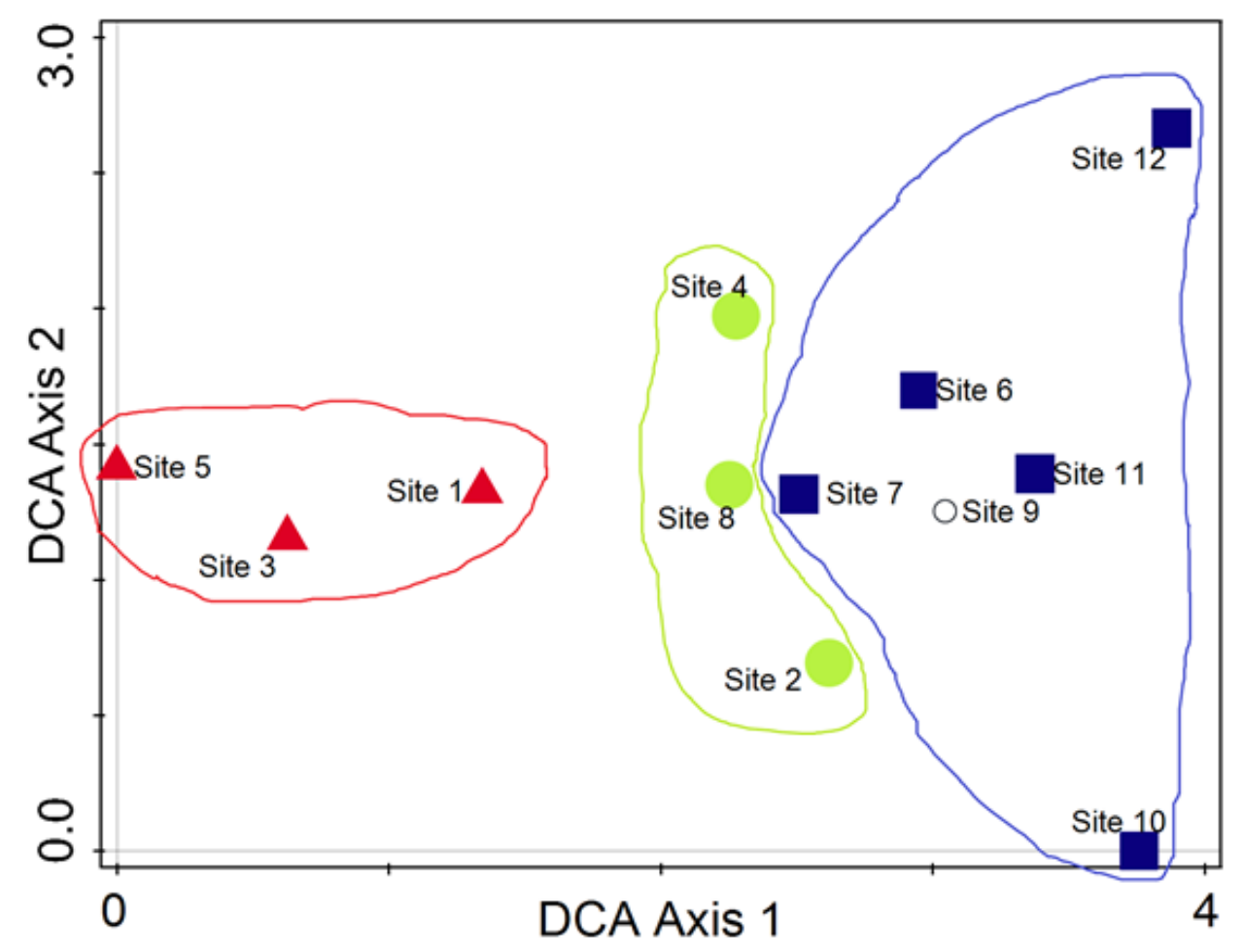

$$
\begin{aligned}
& \text { Key: } \\
& \mathbf{A}=\text { Sites of Olea-Dodonea-Micromeria } \text { association } \\
& =\text { Sites of Pinus-Oxalis-Dactylis association } \\
& \mathbf{\square}=\text { Sites of Pinus-Diospyrus -Myrsine } \text { association }
\end{aligned}
$$

Figure 3. DCA analysis diagram representing distribution pattern of plant association 


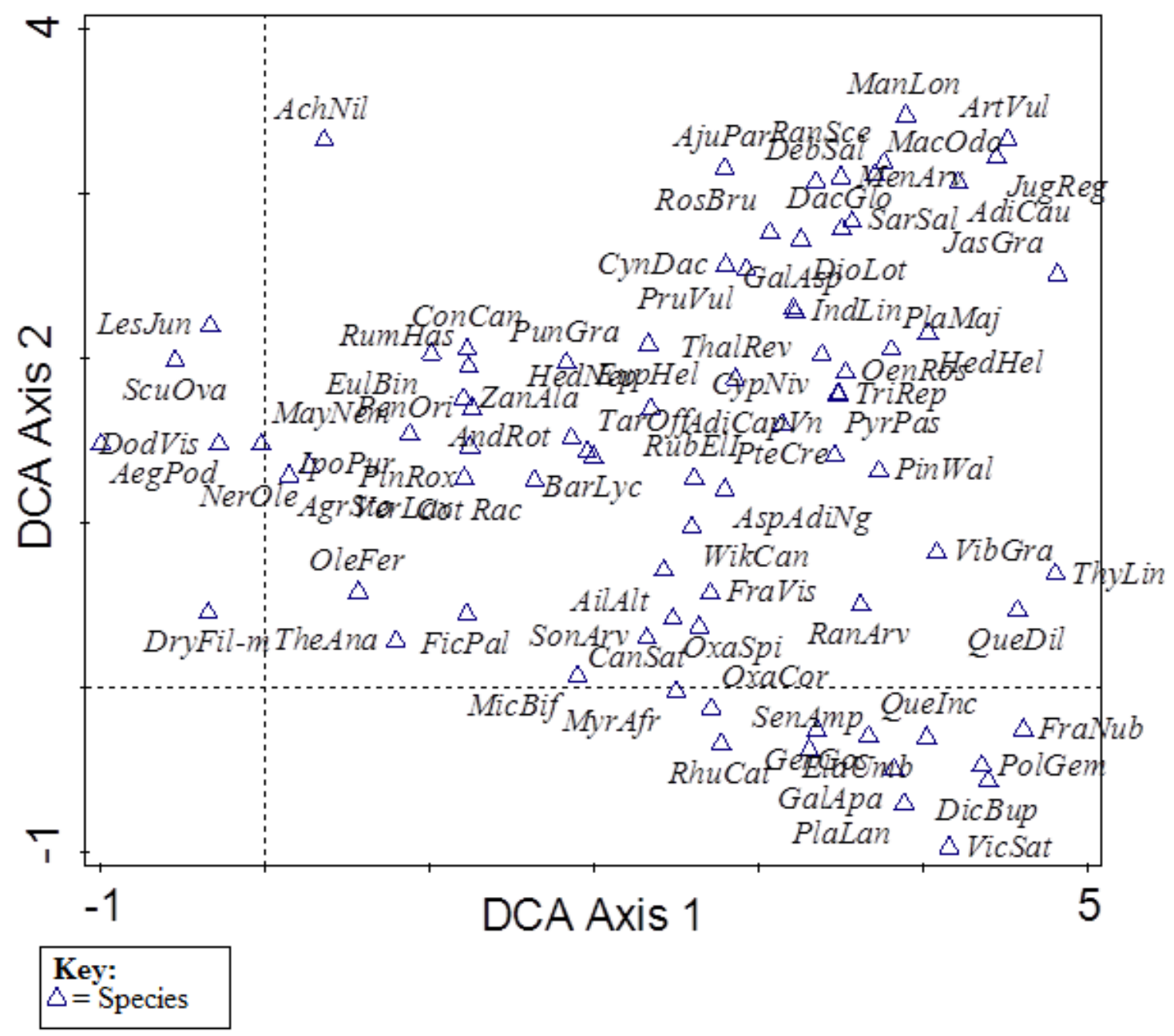

Figure 4. DCA diagram showing distribution of plant species

\section{Canonical correspondence analysis}

Canonical correspondence analysis (CCA) was carried out here to find the relationship between environmental factors and species distribution pattern in different zones of Tehsil Harighel. The high Eigen value of first axis explains high gradient strength along this axis. Nearly half of variation is explained by first two axes therefore remaining two axis were excluded. CCA revealed Altitude and soil physic-chemical properties particularly potassium content, organic matter, electric conductivity and $\mathrm{pH}$ play a significant role in the distribution of plant species while phosphorous and saturation play minor role. The communities harboured at site 1, site 6 and site 7 were under cumulative influence of $\mathrm{pH}$ and electric conductivity. The plant community of sites 2 and site 3 has significant correlation with electric conductivity. The plant communities of site 4 were under cumulative influence of electric conductivity and potassium. Whereas plant communities of site 9 , site 11 and site 12 were under cumulative influence of altitude, potassium and saturation. Site 4 was outlier and not affected by any environmental variable (Fig. 5). Maximum number of species were not affected by any environmental variable as they are located in the center of diagram. E.C play a significant role in grouping of Pinus roxburghii, Punica granatum, Indigofera 


$$
\text { - } 5204 \text { - }
$$

linifolia, Rubus ellipticus, Rumex hastatus. The $\mathrm{pH}$ showed strong correlation with Olea ferruginea, Dodonea viscosa, Dryopteris filix-mas, Silybum marianum and Scutellariaovata. Organic matter plays a significant role in distribution of Jasminum grandiflorum, Dicliptera bupleuroides, Fragaria nubicola and Thymus linearis. Saturation, phosphorus and potassium are important in distribution of Machilus odoratissimus, Rosa brunonii, Cyprus niveus, Urticadioca, Scutellaria chamaedrifolia, Mentha arvensi and Mantha longifolia (Fig. 6).

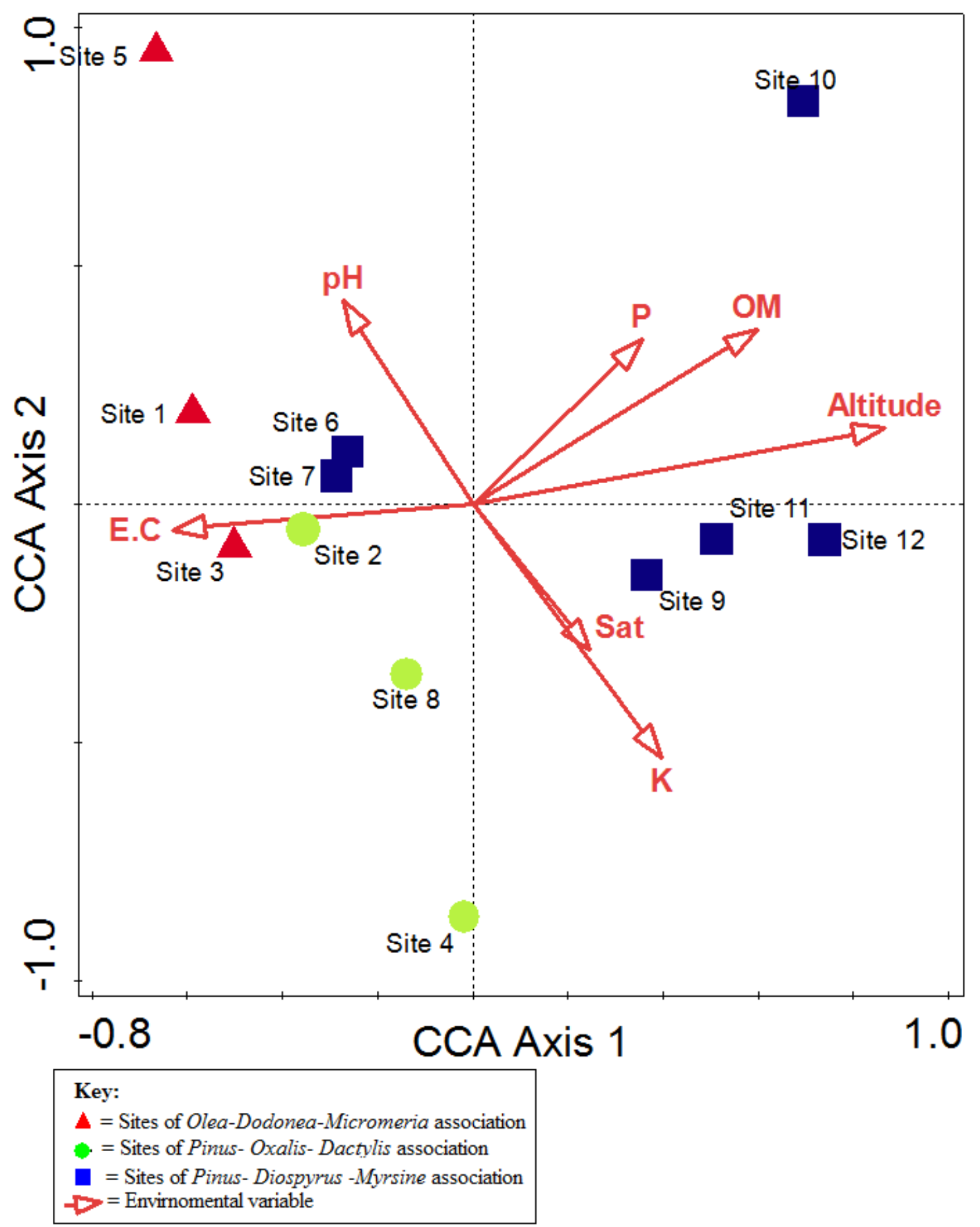

Figure 5. CCA biplot diagram showing distribution of sites along the environmental variable 


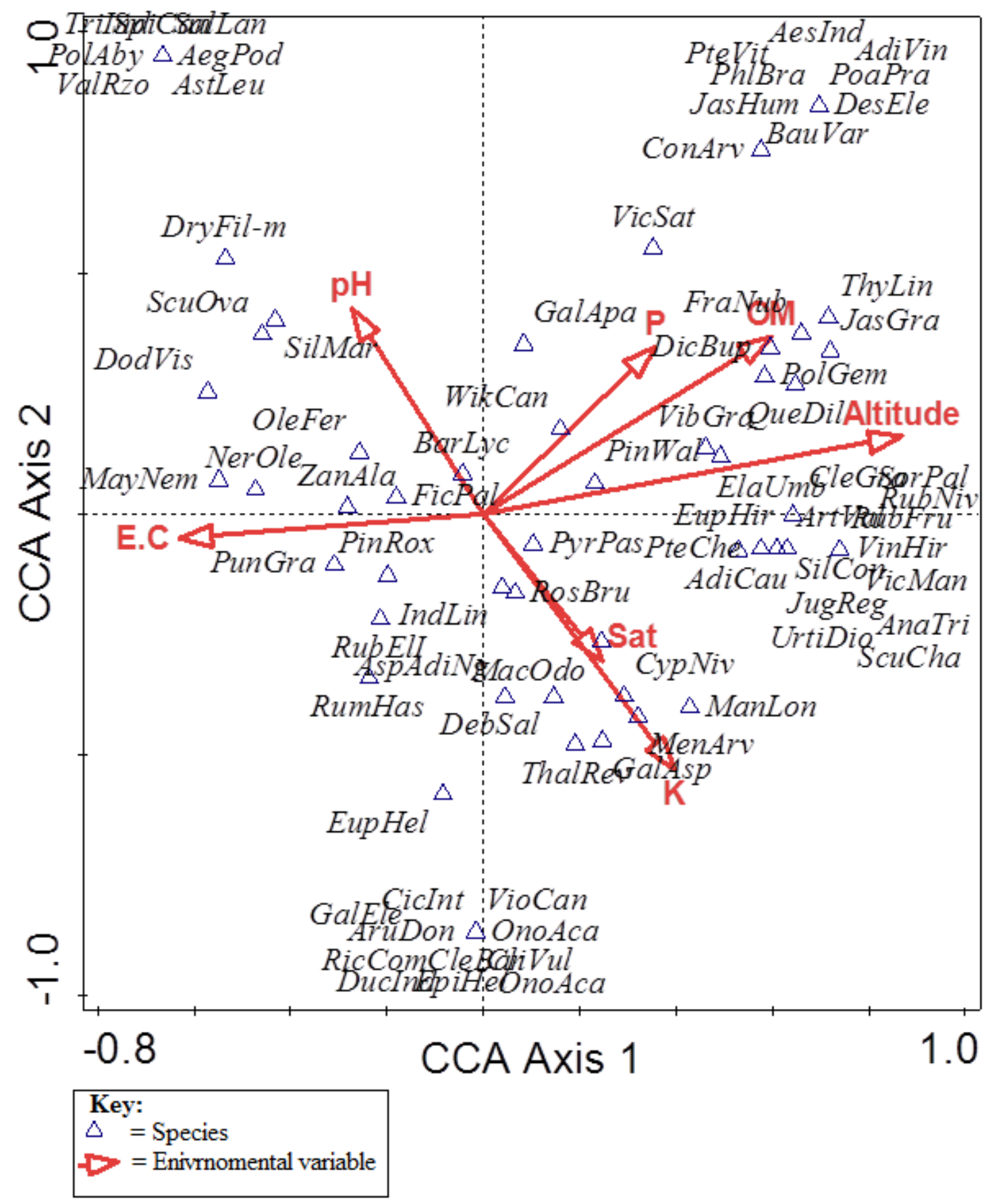

Figure 6. CCA biplot diagram showing distribution of plant species along the environmental variable

\section{Species diversity and its associated components}

The average Shannon diversity was 3.01 and ranged between 2.49 and 3.82. The average diversity was low $(\mathrm{H}=2.55)$ in Olea-Dodonea-Micromeria association and high $(\mathrm{H}=3.29)$ in Pinus-Diospyrus-Myrsine. The average species richness was 4.90 ranged between 3 and 7.01. The richness was low $(\mathrm{R}=3.24)$ in Olea-DodoneaMicromeria association and high $(\mathrm{R}=5.50)$ in Pinus-Oxalis-Dactylis association. The altitude was significantly positively correlated with diversity and richness and explains 
$21 \%$ variation in diversity and $19 \%$ in richness. Average Evenness was 0.63 ranges between 0.46-0.72. Average equitability was 0.86 and ranged between 0.93-0.91. All the forest stands was immature as the maturity value is less than $60 \%$ (Table 2).

Table 2. Diversity and its components recorded from Tehsil Harighel

\begin{tabular}{|c|c|c|c|c|c|c|c|c|}
\hline Association & Communities & $\begin{array}{l}\text { Altitude } \\
\text { (m) }\end{array}$ & $\begin{array}{l}\text { Species } \\
\text { number }\end{array}$ & $\begin{array}{l}\text { Diversity } \\
\text { (H) }\end{array}$ & $\begin{array}{c}\text { Richness } \\
\text { (R) }\end{array}$ & $\begin{array}{c}\text { Evenness } \\
\text { (G) }\end{array}$ & $\begin{array}{l}\text { Equitability } \\
\text { (E) }\end{array}$ & $\begin{array}{l}\text { Maturity } \\
\text { (M) }\end{array}$ \\
\hline \multirow{4}{*}{$\begin{array}{c}\text { Olea- } \\
\text { Dodonea- } \\
\text { Micromeria }\end{array}$} & $\begin{array}{c}\text { Olea- } \\
\text { Dodonaea- } \\
\text { Pinnisetum }\end{array}$ & 980 & 17 & 2.49 & 3.00 & 0.715 & 0.88 & 33.92 \\
\hline & \begin{tabular}{|c|} 
Olea- \\
Micromeria- \\
Dodoneae \\
\end{tabular} & 1165 & 23 & 2.62 & 3.55 & 0.63 & 0.85 & 31.39 \\
\hline & \begin{tabular}{|c|} 
Olea- \\
Aegopodium- \\
Dryopteris \\
\end{tabular} & 1340 & 21 & 2.54 & 3.17 & 0.60 & 0.83 & 37.39 \\
\hline & & & & 2.55 & 3.24 & 0.64 & 0.85 & 34.23 \\
\hline \multirow{4}{*}{$\begin{array}{l}\text { Pinus- } \\
\text { Oxalis- } \\
\text { Dactylis }\end{array}$} & $\begin{array}{c}\text { Dactylis- } \\
\text { Pinus-Myrsine }\end{array}$ & 1079 & 34 & 2.74 & 4.93 & 0.47 & 0.78 & 30.44 \\
\hline & $\begin{array}{c}\text { Pinus-Olea- } \\
\text { Galium }\end{array}$ & 1240 & 48 & 2.97 & 7.01 & 0.71 & 0.91 & 24.78 \\
\hline & $\begin{array}{l}\text { Pinus-pinus- } \\
\text { Wikstroemia }\end{array}$ & 1658 & 28 & 2.97 & 4.56 & 0.72 & 0.90 & 35.92 \\
\hline & & & & 2.89 & 5.50 & 0.63 & 0.86 & 30.38 \\
\hline \multirow{7}{*}{$\begin{array}{l}\text { Pinus- } \\
\text { Diospyrus- } \\
\text { Myrsine }\end{array}$} & $\begin{array}{c}\text { Pinus- } \\
\text { Oenethera- } \\
\text { Trifolium }\end{array}$ & 1450 & 30 & 3.82 & 4.50 & 0.56 & 0.83 & 34.55 \\
\hline & $\begin{array}{c}\text { Pinus- } \\
\text { Fragaria- } \\
\text { Sarcococca }\end{array}$ & 1554 & 38 & 2.96 & 5.30 & 0.55 & 0.83 & 40.61 \\
\hline & $\begin{array}{l}\text { Diospyros- } \\
\text { Pinus }\end{array}$ & 1760 & 40 & 3.19 & 5.32 & 0.67 & 0.88 & 41.05 \\
\hline & $\begin{array}{l}\text { Pinus- } \\
\text { Quercus- } \\
\text { Galium } \\
\end{array}$ & 1864 & 35 & 3.20 & 5.64 & 0.65 & 0.881 & 32.95 \\
\hline & $\begin{array}{c}\text { Pinus- } \\
\text { Micromeria- } \\
\text { Quercus }\end{array}$ & 1964 & 36 & 3.33 & 6.23 & 0.64 & 0.88 & 41.42 \\
\hline & $\begin{array}{l}\text { Pinus- } \\
\text { Adiantum- } \\
\text { Quercus }\end{array}$ & 2052 & 43 & 3.27 & 5.61 & 0.65 & 0.88 & 34.49 \\
\hline & & & & 3.29 & 5.43 & 0.62 & 0.87 & 37.52 \\
\hline
\end{tabular}

\section{Discussion}

Plant associations reflect the environmental condition under which they develop (Malik, 1986; Ilyas et al., 2015). The climate of Tehsil Harigal is of subtropical and 
temperate type (Shaheen et al., 2011a,b; Malik and Malik, 2012) but marked difference in microclimatic, topographic and edaphic factors lead to the establishment of three different plant association with respect to the floristic element and micro environmental conditions. The spatial distribution of plant species in these associations were controlled by different environmental factor like altitude, topography and physcio-chemical properties of soil. The micro gradient established due to variation and interaction among these factors result in different type of vegetation (Hanson and Churchill, 1965) DCA and CCA analysis clearly reflect that altitude and phsico-chemical properties of soil are the main governing factor in the distribution pattern of plant species. This was strongly supported by Ahmad et al. (2009); Shaheen et al. (2012), Amjad et al. (2014 b), Ilyas et al., (2015), Rahman et al. (2016) and Sadia et al. (2017). The large scale pattern in species distribution and physiognomy is mainly governed by climate but physicochemical properties of soil also govern the distribution pattern on local or micro level (Bakkenes et al., 2002). Climate can be characterized by different variable mainly available moisture which mainly determines the distribution pattern (Leeman and Cramer, 1991). The moderate rainfall and high temperature of Tehsil Harigal result in the stratified forests. Soil is key factor that play important role in selection of plant by bringing evolutionary changes (Barbour et al., 1980). The vegetation of particular area has strong relationship with the soil (Ali et al., 2004). The physico- chemical properties are directly related to soil depth which play key role in establishment of plant association or communities (Khan et al., 2013) The Electrical conductivity of soil of Olea-Dodonea-Micromeria association was high whereas organic matter, $\mathrm{pH}$, available moisture nutrient content as compared to Pinus-Oxalis-Dactylis association and PinusDiospyros-Myrsine association which result in establishment of different vegetation type. Slight differences in the available nutrients are positively correlated with variations in community structure (Noor and Khatoon, 2013).

Species diversity is reflection of the health and productivity of ecosystem. Diversity was low in the investigated area due to heavy grazing, deforestation, road construction and fuel wood extraction etc. (Ram et al., 2004; Kumar and Bhatt, 2006). The species diversity and richness was high in Pinus-Diospyrus-Myrsine association which might be due to number of coexisting and interacting plant species having overlapping niche (Saxena and Singh, 1982). Moreover the site of higher altitude (Pinus-DiospyrusMyrsine association) are difficult to access by local inhabitant which also resulting high species diversity and richness. The species richness and diversity is positively correlated with altitude. Our findings are in accordance with Malik and Malik (2012) who reported positive correlation between diversity and altitude which might be due to increase in humidity. Differences in altitudes, aspects and slopes result in variation in species diversity and association types. The plant communities harboured at North facing slopes have high diversity with thick vegetation because of high moisture content on southern slope as compared to north facing slope. The same results were obtained by Khan et al. (2011); Amjad et al. (2014a) and and Haq et al. (2015). Plant growth and survival can be affected by amount of water runoff and infiltration which is dependent upon position and smoothness of slope. Aspect and steepness of slope affect the amount of solar radiation which in turn affect temperature on the ground surface (Sukopp and Werner, 1983) and the amount and type of soil accumulated (Monsen et al., 2004). The evenness and equitability is intermediate due to the long term stable climatic condition and uniform pattern of species distribution in majority of plant communities in Harigal. The maturity index reflects immature forest structure throughout investigated area because 
plant species were less adapted to microclimate. The natural balance of plant communities was disturbed due to high anthropogenic pressures which further enhance this pattern (Saxena and Singh, 1982; Shaheen et al., 2011a).

\section{Conclusion}

The current study suggests that environmental gradient has profound influence on the distribution of plant species in Tehsil Harighel and association of plant change due to change in environmental variable. Further low value of species diversity and associated indices reflects deteriorating forest structure due to immense anthropogenic pressure in the form of intense grazing, severe deforestation for fuel, fodder and construction purpose, trampling and road construction etc. Therefore immediate conservations measures are needed to preserve the rich plant species diversity of these degraded forests. Land use mapping and vegetation assessment using modern software like ERDAS, arc GIS and NDVI, and are also recommended.

\section{REFERENCES}

[1] Ahmad, S. S., Fazal, S., Valeem, E. E., Khan, Z. I., Sarwar, G., Iqbal, Z. (2009): Evaluation of ecological aspects of roadside vegetation around Havalian city using multivariate techniques. - Pak. J. Bot. 41(1): 53-60.

[2] Ahmad, S. S., Khan, S., Erum, S. (2013): Multivariate analysis of roadside vegetation along motorway (m-1), Pakistan. - Pak. J. Bot. 45(1): 49-53.

[3] Ali, M., Ahmad, T., Rashid, A. (2004): Phytosociological synthesis as inferred from soil analysis of some Industrial areas of the Punjab. - Asian J. Pl. Sci. 3(3): 320-324.

[4] Amjad, M. S., Arshad, M., Chaudhari, S. K. (2014a): Structural diversity, its components and regenerating capacity of lesser Himalayan forests vegetation of Nikyal valley District Kotli (A.K), Pakistan. - Asi. Pac. J. Trop. Med. 7(Suppl 1): 454-460.

[5] Amjad, M. S., Arshad, M., Rashid, A., Chaudhari, S. K., Malik, N. Z., Fatima, S. (2014b): Examining relationship between environmental gradients and Lesser Himalyan forest vegetation of Nikyal valley, Azad Jammu and Kashmir using ordination analysis. Asi. Pac. J. Trop. Med. 7(Suppl 1): 610-616.

[6] Anderson, M. J., Ellingsen, K. E., McArdle, B. H. (2006): Multivariate dispersion as a measure of beta diversity. - Ecology Letters 9: 683-693.

[7] Anonymous (2007): AJK at a Glance. - Pakistan Planning \& Development Department, Government of Azad Jammu and Kashmir, Muzaffarabad.

[8] Anonymous (2009): The Normals of Climatic Data of Azad Jammu \& Kashmir. Pakistan Pakistan Meteorological Department, Islamabad.

[9] Bakkenes, M., Alkemade, J. R., Ihle, F., Leemans, R., Latour, J. B. (2002): Assessing effects of forecasted climate change on the diversity and distribution of European higher plants for 2050. - Global Change Biology 8(4): 390.

[10] Barbour, M. G., Burk, J. H., Pitts, W. D. (1980): Terrestrial Plant Ecology. - The Benjamin, California.

[11] Bergmeier, E. (2002): The vegetation of the high mountains of Crete. A revision and multivariate analysis. - Phytocoenologia 32: 205-249.

[12] Bokhari, T. Z., Liu, Y., Ahmed, M., Siddiqui, M. F., Li, Q., Khan, Z. U., Malik. S. A. (2016): Multivariate analysis of some pine forested areas of Azad Kashmir, Pakistan. Pak. J. Bot. 48(1): 105-113.

[13] Curtis, J. T., McIntosh, R. P. (1950): The Interrelations of Certain Analytic and Synthetic Phytosociological Characters. - Ecology 31: 434-455. 
[14] Daubenmire, R. (1968): Plant Communities. A textbook of Plant Synecology. - Harper \& Row, New York.

[15] Dufrene, M., Legendre, P. (1997): Species assemblages and indicator species: the need for a flexible asymmetrical approach. - Ecol. Monogr. 67: 345-366.

[16] Ellenberg, H. (1996): Vegetation Mitteleuropas mit den Alpen (5th edition). - Ulmer, Stuttgart.

[17] Gairola, S., Rawal, R. S., Todaria. N. P. (2008): Forest vegetation patterns along an altitudinal gradient in sub-alpine zone of west Himalaya, India. - African Journal of Plant Science 2(6): 042-048.

[18] Gallardo-Cruz, J. A., Perez-Garcia, E. A., Meave, J. A. (2009): Diversity and vegetation structure as influenced by slope aspect and altitude in a seasonally dry tropical landscape. - Landscape Ecol. 24: 473-482.

[19] Gardner, T. A., Barlow, J., Chazdon, R., Ewers, R. M., Harvey, C. A., Peres. C. A. (2009): Prospects for tropical forest biodiversity in a human-modified world. - Ecology Letters 12: 561-582.

[20] Hanson, H., Churchill, E. D. (1965): The Plant Community. - Reinhold, New York.

[21] Haq, F., Ahmad, H., Iqbal, Z. (2015): Vegetation description and phytoclimatic gradients of subtropical forests of Nandiar Khuwar catchment District Battagram. - Pak. J. Bot. 47(4): 1399-1405.

[22] Hussain, F. (1989): Field and Laboratory Manual of Plant Ecology. - University Grants Commission, Islamabad.

[23] Hussain, F., Illahi, I (1991): Ecology and Vegetation of Lesser Himalayan Pakistan. Bot. Dept. Uni. of Peshawar.

[24] Ilyas, M., Qureshi, R., Raja, N. A., Munir, M., Haq, Z. U. (2015): Vegetation analysis of Kabal valley, District Swat, Pakistan using multivariate approach. - Pak. J. Bot. 47(SI): 77-86.

[25] Jackson, M. L. (1962): Significance of Kaolinite Intersalation in Clay Mineral Analysis. 9th Conf. Clays and Clay Minerals, Pergamon Press, New York, NY. pp. 424-430.

[26] Kent, M., Coker, P. (2012): Vegetation Description and Data Analysis: A Practical Approach. - John Wiley and Sons Ltd, United Kingdom.

[27] Khan, N., Shaukat, S. S., Ahmed, M., Siddiqui, M. F. (2013): Vegetation-environment relationships in the forests of Chitral district Hindukush range of Pakistan. - J. Forest. Res. 24(2): 205-216.

[28] Khan, S. M., Harper, D. M., Page, S., Ahmad, H. (2011): Species and community diversity of vascular flora along environmental gradient in Naran Valley: a multivariate approach through Indicator Species Analysis. - Pak. J. Bot. 43: 2337-2346.

[29] Kumar, M., Bhatt, V. (2006): Plant biodiversity and conservation of forests in foot hills of Garhwal Himalaya. - Lyonia 11(2): 43-59.

[30] Leemans, R., Cramer, W. (1991): The I.I.A.S.A. Climate Database for Mean Monthly Values of Temperature, Precipitation and Cloudiness on a Terrestrial Grid. - I.I.A.S.A, Laxenburg.

[31] Malik, Z. H. (1986): Phytosociological Study on the Vegetation of Kotli Hills, Azad Kashmir. - M.Phil. Thesis, Universtiy of Pesawar.

[32] Malik, Z. H., Malik, N. Z. (2012): High altitude forest composition diversity and its component in a part of Ganga chotti and bedori hills district bagh. Azad Jammu and Kashmir, Pakistan. - AGD Landscape Environ. 6(1): 31-40.

[33] Margalef, R. (1958): Information theory in ecology. - General Systems 3: 36-71.

[34] McCune, B., Grace, J. B. (2002): Analysis of Ecological Communities. - MjM Software, Gleneden Beach, Oregon.

[35] McCune, B., Mefford, M. J. (1999): PC-ORD: Multivariate Analysis of Ecological Data; Version 4 for Windows (User's Guide). - MjM Software Design, Gleneden Beach, Oregon. 
[36] McCune, B., Mefford, M. J. (2005): Multivariate Analysis of Ecological Data (PCORD Version 5.10) - MjM Software, Gleneden Beach, Oregon.

[37] Monsen, S. B., Stevens, R., Nancy, L. (2004): Restoring western ranges and wild lands. Gen. Tech. Rep. Fort Collins, Co, U.S. Department of Agriculture, Forest Service. Rocky Mountain Research Station 136(1): 1-294.

[38] Noor, A., Khatoon, S. (2013): Analysis of vegetation pattern and soil characteristics of Astore valley GilgitBaltistan. - Pak. J. Bot. 45(5): 1663-1667.

[39] Pichi-Sermolli, R. (1948): An index of establishing degree of maturity in plant community. - J. I. Col. 38: 85-90.

[40] Rahman, A. U., Khan, M. S., Khan, S., Hussain, S., Rahman, I., Iqbal, Z., Ijaz, F. (2016): Ecological assessment of plant communities and associated edaphic and topographic variables in the Peochar Valley of the Hindu Kush Mountains. - International Mountain Society 36(3): 332-341.

[41] Ram, J., Kumar, A., Bhatt, J. (2004): Plant diversity in six forest types of Uttaranchal, Central Himalaya, India. - Curr. Sci. 86(7): 975-978.

[42] Ruiz, D., Moreno, H. A., Gutierrez, M. E., Zapata, P. A. (2008): Changing climate and endangered high mountain ecosystems in Colombia. - Sci. of the Total Environ. 398: 122-132.

[43] Sadia, S., Jin-Tun, Z., Tariq, A., Bai, X., Sheday, A. A., Cao, K., Mazarii, P., Sidra, I., Latif, A. (2017): Species diversity, vegetation pattern and conservation of Gentiana macrophylla Pall. communities in Dongline Mountain meadow, Beijing, China. - Pak. J. Bot. 49(5): 1725-1734.

[44] Saxena, A. K., Singh, J. S. (1982): A phytosociological analysis of woody species in forest communities of a part of Kumaun Himalaya. - Vegetatio 50(1): 3-22.

[45] Scherrer D, Korner, C. (2010): Infra-red thermometry of alpine landscapes challenges climatic warming projections. - Global Change Biology 16: 2602-2613.

[46] Schuster, B., Diekmann, M. (2005): Species richness and environmental correlates in deciduous forests of northwest Germany. - Forest Ecology and Management 206: 197205.

[47] Shaheen, H., Ullah, Z., Khan, S. M., Harper, D. M. (2012): Species composition and community structure of western Himalayan moist temperate forests in Kashmir. - Forest Ecol. Manag. 278: 138-145.

[48] Shaheen, H., Qureshi, R. A., Shinwari, Z. K. (2011a): Structural diversity, vegetation dynamics and anthropogenic impact on lesser Himalayan subtropical forests of Bagh district, Kashmir. - Pak. J. Bot. 43(4): 1861-1866.

[49] Shaheen, H., Khan, S. M., Harper, D. M., Ullah, Z., Qureshi, R. A. (2011b): Species diversity, community structure, and distribution patterns in western Himalayan alpine pastures of Kashmir, Pakistan. - Mountain Res. \& Development 31(2): 153-159.

[50] Shannon, C. E., Wiener, W. (1949): The Mathematical Theory of Communication. University of Illonois Press, Urgana.

[51] Sheldon, A. L. (1969): Equitability indexes: dependences on the species account. - Ecol. 50(1): 466-467.

[52] Shoukat, S. S., Kheri, M. A., Khan, M. A. (1978): The relationships amongst dominance, diversity and community maturity in desert vegetation. - Pak. J. Bot. 10(2): 183-196.

[53] Shrestha, B. B., Jha, P. K. (2009): Habitat range of two alpine medicinal plants in a transHimalayan dry valley, central Nepal. - Journal of Mountain Science 6: 66-77.

[54] Siddiqui, F. M., Ahmed, M., Shaukat, S. S., Khan, N. (2010): Advance multivariate techniques to investigate vegetation-environmental complex of pine forests of moist area of Pakistan. - Pak. J. Bot. 42(SI): 267-293.

[55] Sukopp, H., Werner, P. (1983): Urban Environments and Vegetation. - In: Holzner, W. et al. (ed.) Man's Impact of Vegetation. Junk Publisher, The Hague.

[56] Tavili, A. Jafari, M. (2009): Interrelations between plants and environmental variables. Int. J. Environ. Res. 3: 239-246. 
[57] Ter Braak, C. J., Prentice, I. C. (1988): A theory of gradient analysis. - Adv. Ecol. Res. 18: 271-317.

[58] Ter Braak, C. J. F., Smilauer, P. (2002): CANOCO Reference Manual and CanoDraw for Windows User's Guide: Software for Canonical Community Ordination (version 4.5). www.canoco.com. Microcomputer Power, Ithaca, NY.

[59] Vetaas, O. R. (2000): Comparing species temperature response curves: Population density versus second-hand data. - Journal of Vegetation Science 11: 659-666.

[60] Vetaas, O., Grytnes, J. A. (2002): Distribution of vascular plant species richness and endemic richness along the Himalayan elevation gradient in Nepal. - Global Ecology \& Biogeography 11: 291-301.

[61] Whittaker, R. H. (1977): Evolution of species diversity in land plant communities. Evolutionary Biology 10: 1-67.

[62] Willoughby, M. G., Alexander, M. J. (2000): A Range Condition Dilemma. - Rangelands 22(1): 23-26.

[63] Willoughby, M. G., Alexander, M. J. (2005): Long-Term Trends in Rangeland Health of the Rough Fescue Ecological Site in the Montane Subregion. - Alberta Sustainable Resource Development. Public Lands and Forest Division, Edmonton. 